Projets

de paysage

\section{Projets de paysage}

Revue scientifique sur la conception et l'aménagement de l'espace

19 | 2018

L'imagerie du paysage

Marcher ensemble : expérience dans le paysage, de la spatialité à l'expérience commune

Walking Together: An Experience in the Landscape, From Spatiality to a

Common Experience

\title{
Ekaterina Shamova
}

\section{CpenEdition}

\section{Journals}

Édition électronique

URL : http://journals.openedition.org/paysage/425

DOI : $10.4000 /$ paysage.425

ISSN : 1969-6124

Éditeur :

École nationale supérieure du paysage de Versailles-Marseille, Institut national des sciences appliquées Centre Val de Loire - École de la nature et du paysage, École nationale supérieure d'architecture et de paysage de Bordeaux, École nationale supérieure d'architecture et de paysage de Lille, Agrocampus Angers

\section{Référence électronique}

Ekaterina Shamova, « Marcher ensemble : expérience dans le paysage, de la spatialité à l'expérience commune », Projets de paysage [En ligne], 19 | 2018, mis en ligne le 01 décembre 2018, consulté le 28 novembre 2019. URL : http://journals.openedition.org/paysage/425;DOI : 10.4000/paysage.425

Ce document a été généré automatiquement le 28 novembre 2019.

Projets de paysage 


\section{Marcher ensemble : expérience dans le paysage, de la spatialité à l'expérience commune}

Walking Together: An Experience in the Landscape, From Spatiality to a Common Experience

Ekaterina Shamova

\section{Esquisse générale de la démarche et état de l'art sur la question}

1 Cet article présente l'un des aspects traités dans une thèse en cours, à savoir les projets de marche collective proposés par les artistes et/ou chorégraphes. Ces projets suivent un certain protocole, et se déroulent dans des paysages divers. Ils engagent les participants dans une expérience avec l'espace environnant et avec les autres par l'itinérance, par le travail sur le corps, sur les sens, sur l'imaginaire. Je vais évoquer deux projets auxquels j'ai participé. Ce sont les Promenades blanches de l'artiste Mathias Poisson et du chorégraphe Alain Michard et sa version expérimentée en mai 2017, et le projet Attention à la marche ! qui fut coproduit en juillet 2017 par Le Bruit du frigo ${ }^{1}$ (Bordeaux) et le Bureau des guides (Marseille) avec la participation de Mathias Poisson, Robin Decourcy, Laurent Petit et le collectif La Folie Kilomètre.

2 Les pratiques et les démarches de certains artistes semblent se développer avec, en parallèle, les volontés de certains acteurs dans les domaines de l'urbanisme, de l'aménagement, du développement du territoire ou des politiques culturelles d'intégrer la dimension de la marche, de l'itinérance dans leurs champs respectifs. Les raisons pour cela sont diverses et vont au-delà de la simple volonté de proposer un événement artistique. La marche est convoquée pour révéler la ville ou le quartier autrement, pour relier le territoire, pour apporter une nouvelle approche à une problématique urbaine, ou pour mettre en lumière les spécificités d'un lieu du point de vue de son contexte 
social ou géographique. Parmi les exemples artistiques les plus récents, nous pouvons mentionner le projet " Académie de la marche » du Magasin des Horizons de Grenoble en 2017-2018, les initiatives du Bureau des guides autour du sentier GR2013, le projet "Les Marches" dans le cadre de la biennale Panoramas à Bordeaux au mois de mai 2018. Mon questionnement élargi concernera la compréhension de telles dynamiques et de leur attachement territorial. Il semble qu'une certaine vision des manières de vivre le paysage et de se représenter le territoire soit inhérente à ces initiatives. Comment les intentions des commanditaires et des artistes façonnent-elles l'expérience vécue, à la fois individuelle et commune, du territoire?

3 Autre chose à souligner est une attention portée par les chercheurs aux pratiques de la danse et de la chorégraphie qui s'emparent de la marche. Ces recherches visent à comprendre en quoi les «expériences chorégraphiques hodologiques [...] reposant sur [1]es savoirs du mouvement et de l'espace » (Perrin 2017) peuvent renouveler le rapport qu'un individu entretient avec le monde. De l'autre côté, les figures des artistesmarcheurs ${ }^{2}$ semblent être étudiées de manière autonome par rapport à ces pratiques (ibid.). Dans ma recherche ultérieure, je voudrais exploiter le lien et la continuité existant entre les pratiques des artistes-marcheurs et celles des chorégraphes qui proposent les promenades à leur public. Cet écart d'ordre méthodologique a été exprimé, par exemple, lors des journées d'études sur les promenades chorégraphiques et sonores organisées par Julie Perrin et Nicolas Dornin dans le cadre du programme "Arts, écologies, transitions" du Labex Arts- $\mathrm{H} 2 \mathrm{H}$ au Centre national de la danse (Pantin) en janvier 2018. Ces journées ont présenté les recherches menées sur les promenades, en se focalisant sur divers aspects - sensibles, corporels, esthétiques, paysagers. Les questionnements évoqués touchaient la dimension hodologique de tels projets et l'expérience convoquée, les manières dont ceux-ci s'inscrivaient dans le paysage, à travers le mouvement, et les façons de s'en rendre compte ${ }^{3}$. Pour ma part, je voudrais me concentrer sur le lien entre le paysage et son expérience dans sa dimension immédiate, à la fois individuelle et collective. Ceci en pensant les manières dont cette expérience peut être envisagée (commanditaires, artistes, expériences projetées) et en quoi celles-ci peuvent révéler et transformer le territoire.

4 Certains projets auxquels je m'intéresse ont fait l'objet de recherches. Les projets de Mathias Poisson ont été étudiés par Julie Perrin, chercheuse en danse. Elle a traité le lien entre l'expérience et les manières de la représenter, ainsi que la question de la trace. Mathilde Christmann, doctorante au LACTH, a étudié la partition chez Mathias Poisson, en lien avec sa recherche sur Lawrence Halprin. Élise Olmédo, docteure en géographie, a travaillé sur des cartes sensibles de l'artiste. Mon angle d'approche concernant ce projet, et les autres projets qui m'intéressent, se focalise sur la spatialité individuelle des participants et sur les manières dont l'expérience commune se développe.

\section{Quelles hypothèses pour les projets en question? Présupposé paysager}

Mon intérêt porte sur certains projets artistiques proposant des marches collectives dans le paysage et engageant un groupe de marcheurs. Quelles sont alors les qualités d'une présence dans le paysage, individuellement et dans un groupe ? Comment est-il possible de définir et de décrire l'expérience du participant de ces projets, dans la 
multitude de ses aspects - kinesthésiques, sensoriels, corporels, imaginaires ? Comment interagit-elle avec celle d'autrui ?

La problématique de cette recherche s'axe sur l'expérience vécue dans les projets de la marche, qui serait à la fois propre à chacun, et en même temps ferait surgir quelque chose en groupe. Ma première hypothèse est la suivante : il serait possible de discerner plusieurs dimensions qui révéleraient l'expérience des participants à de tels projets kinesthésiques, corporelles, sensorielles, proprioceptives, imaginaires, et ce, dans l'immédiateté des rapports avec le monde. En cela, cette expérience pourrait correspondre au " contact naif avec le monde » selon Maurice Merleau-Ponty (1995, p. 7). La personne structurerait l'espace par ses mouvements, par son corps, par ses sensations, par son imagination, par sa mémoire. Se poserait donc une question au sujet de la spatialité qui reflète la situation immédiate du sujet dans l'espace-temps précis et les sensations de celui-ci.

7 Ensuite, puisque la marche s'effectue en groupe, il y aurait un passage de la spatialité individuelle à quelque chose qui serait commun dans cette présence collective. Ceci adviendrait dans l'immédiateté de rapports se formant avec l'espace, les autres, les expériences proposées, les paysages traversés. L'aspect collectif de cette expérience et les manières dont elle est envisagée (par les commanditaires, par les artistes) m'ont permis de rapprocher la question de l'expérience et celle paysagère. Les passerelles existeraient entre les manières d'être au monde proposées par certaines pratiques, le vécu immédiat des participants, et les intentions territoriales visées par les commanditaires ${ }^{4}$. De ce fait, je propose l'hypothèse suivante : il existerait un lien entre la question de la spatialité dans ses aspects individuels et collectifs, comme présence dans le paysage, et le projet élargi lié aux manières de vivre et de se déplacer sur un territoire donné. De quelle manière les notions de territoire et de paysage s'articulentelles dans le cas des projets étudiés? Le territoire serait à comprendre au sens, plus restreint, géographique et politique, et inclurait les visées institutionnelles et conceptuelles pour les projets en question. Je propose donc un supposé selon lequel les manières d'envisager et de vivre un territoire concret, par un projet artistique spécifique convoqué dans un cadre institutionnel, seraient en dialogue avec les façons de vivre une expérience avec le paysage, à la fois individuellement et collectivement. Je m'arrêterai sur cette hypothèse plus longuement. Ainsi, de quelle manière le paysage se révèle-t-il dans les projets en question? Quels seraient les indices dans l'expérience immédiate du participant qui permettraient de caractériser sa relation avec le paysage ? Comment l'expérience proposée à un groupe de marcheurs rentrerait-elle en dialogue avec le projet pour le territoire traversé ? Dans la partie qui suivra, j'aborderai quelques éléments portant sur la vision contemporaine du paysage qui, selon mon hypothèse, pourraient véhiculer le lien entre l'expérience, la présence collective et le territoire.

\section{Entre expérience et paysage. Quelques approches théoriques}

8 Mes bases théoriques associent quelques notions. Ma réflexion sur la question de l'expérience immédiate du sujet et sa relation au monde s'appuie sur la notion de la "spatialité » telle qu'elle est explicitée par Erwin Straus, neuropsychiatre allemand (Straus, 2000). Toutefois, la notion de spatialité caractérise une relation au monde du 
point de vue individuel. Il est donc nécessaire de comprendre comment passer d'une expérience individuelle à une expérience collective. Pour étayer cette transposition, je m'adresserai à la notion de "correspondance " (Ingold, 2013) de l'anthropologue britannique Tim Ingold. En supposant ensuite que l'expérience au sein des projets de la marche soit ancrée dans une certaine interprétation de paysage et dans une vision particulière du territoire et des manières de le vivre, j'essaierai de proposer un cadre de réflexion qui embrassera ces deux perspectives. Pour cela, je vais me baser sur les définitions du paysage proposées par le philosophe Jean-Marc Besse (Besse, 2009) en ce qui concerne ses aspects expérientiels et matériels.

9 La spatialité énoncée par Erwin Straus dans son ouvrage datant de 1935 Du sens des sens ${ }^{5}$ désigne l'unité du sentir et du se mouvoir, du sujet sentant dans l'espace-temps et dans l'immédiateté. Le sujet est un être en devenir dans et avec le monde environnant. L'unité du sentir et du se mouvoir est nommée par Straus le "rapport de totalité " (Straus, 2000, p. 293) qui ne peut être tel quel si le sujet est présent au monde, dans l'immédiateté. L'homme se meut avec le monde dans ce rapport de totalité, dans la mesure où il est toujours renouvelé. Une telle définition de l'expérience me permet de me questionner plus particulièrement sur le sentir qui serait individuel. Ce qui doit être souligné dans cette posture, c'est le fait que l'expérience vécue du monde est pour le sujet concret, "pour moi»: "Le hic et nunc est uniquement pour moi, dans mon monde, mais les deux aspects sont la délimitation spécifique de la totalité de ma relation personnelle avec le monde ${ }^{6} »$ (ibid., p. 294). Si je peux, à travers cette notion, analyser l'expérience qui est la mienne ou celle d'un autre participant, la question est : quelle expérience se forme dans un groupe?

Si la spatialité est l'expérience du monde qui est la mienne, de quelle manière puis-je caractériser une présence collective ? Sera-t-elle celle du sentir? Sans toutefois qu'il y ait un lien affirmé entre les propos des deux auteurs, la vision du monde introduite par Tim Ingold pourrait avoir des points de convergence avec la notion de spatialité straussienne. La notion de correspondance qu'il introduit dans son ouvrage datant de 2013 The Making. Anthropology, archaeology, art and architecture (Ingold, 2013) permettrait de passer à l'analyse de l'expérience collective. De manière générale, l'anthropologue considère que le monde est "un processus continuel » (Descola et Ingold, 2014, p. 38-39). Il s'agit pour Ingold de l'existence des matières, plutôt que des objets (entités figées). Il y aurait continuellement des allers-retours entre les choses ; et la vie même serait « un entremêlement qui n'est pas formé pas des points reliés entre eux, mais par des lignes entrecroisées [...] un maillage » (Ingold, 2017). La manière dont les choses interagissent ne serait pas " une imposition d'une forme préconçue sur une substance matérielle brute, mais le fait de dessiner ou faire apparaitre le potentiel immanent d'un monde en devenir. Dans un monde phénoménologique, chaque matériau est un tel devenir, une apparition ${ }^{7} »$ (Ingold, 2013, p. 31). Ainsi, Ingold insiste sur le caractère fluctuant et perméable des matières et sur le rapport dynamique entre les choses. Je retiens cette idée pour pouvoir explorer son lien avec l'expérience en marche dans sa dimension collective, mais aussi avec le paysage. La relation se formerait entre la personne et le monde, ou entre les personnes dans un groupe et le paysage traversé. Je suis amenée à penser qu'elle pourrait corroborer le mode de présence à plusieurs qui serait celui du sentir, dans le cas des projets «Promenades blanches » et "Attention à la marche!». La relation entre la personne et le monde environnant, entre les personnes en groupe, se transformerait dans un rapport 
réciproque incluant les dimensions variables (kinesthésiques, proprioceptives, sensibles, corporelles, imaginaires, spatiales, temporelles) et se développerait en mouvement, en trajet.

11 Il serait question par la suite d'articuler le lien entre l'expérience immédiate du sujet et des sujets en groupe et le paysage dans lequel elle se développe. J'ai insisté précédemment sur un présupposé selon lequel les façons d'envisager et de vivre un territoire individuellement et collectivement pourraient s'articuler avec une vision de paysage ancrée dans son expérience et dans les manières d'y être présent. Dans son ouvrage Le Goût du monde. Exercices de paysage (2009), Jean-Marc Besse a proposé cinq approches différentes quant à la définition du paysage, en fonction des domaines et modes de pensée qui traitent la question du paysage. Une des approches selon laquelle ce dernier serait propice à une expérience phénoménologique m'a semblé en phase avec la réflexion que je mène. Dans cette optique : «[...] le paysage peut être [...] compris et défini comme l'événement de la rencontre concrète entre l'homme et le monde qui l'entoure. Le paysage est avant tout, en ce cas, une expérience » (Besse, 2009, p. 50). Le paysage relève du contact direct de l'homme avec le monde, dans un engagement sensible, corporel et émotionnel ${ }^{8}$. Il serait ancré dans le présent à travers une "rencontre concrète " qui créerait des conditions pour un surgissement d'une certaine expérience du monde. Cette rencontre serait aussi celle qui nous ouvrirait à l'autre, nous ferait « [...] participer au mouvement du monde en un lieu donné » (ibid.). Je retiens cette idée de l'expérience concrète dans un «lieu donné » qui serait, à mon avis, appropriable dans le cas des projets qui sont l'objet de mes analyses. Dans cette formulation, je voudrais revenir à mon hypothèse où le vécu immédiat, à la fois individuel et commun, aurait une relation avec le territoire traversé. Ce territoire serait d'une certaine manière envisagé par les intentions institutionnelles et par les protocoles artistiques qui chercheraient à proposer une façon de vivre le territoire, en groupe, sur une temporalité donnée. Il m'a donc semblé que la vision du paysage qui le considère comme destiné à répondre aux besoins des hommes qui y vivent pourrait soutenir ce propos. Jean-Marc Besse reprend cette veine de réflexion chez l'historien du paysage américain John Brinckerhoff Jackson. Selon lui, le paysage serait un territoire fabriqué et habité par les hommes :

«Il est d'abord une réalité objective, matérielle, produite par les hommes. Tout paysage est culturel, non pas d'abord parce qu'il est vu par une culture, mais d'abord parce qu'il a été produit au sein d'un ensemble de pratiques (économiques, politiques, sociales), et selon des valeurs que d'une certaine manière il symbolise. » (Ibid., p. 34)

12 Selon cette posture, les qualités matérielles et concrètes du paysage sont évoquées. Nous pouvons alors parler des manières dont les hommes façonnent ces données pour que cela puisse "servir une communauté » (ibid., p. 38). Je voudrais alors tracer des parallèles entre les deux visions de paysage ici présentées. Elles permettraient d'abord de penser les manières dont une expérience peut advenir à travers le contact de la personne avec le monde. Puis, l'expérience, à la fois individuelle et collective, pourrait s'inscrire dans le projet plus large de construction d'un territoire, sur le temps d'un projet artistique, à partir des intentions exprimées par les commanditaires, des protocoles des artistes et de leurs manières de configurer l'expérience. Comment pouvons-nous inscrire l'expérience du paysage dans son aspect immédiat, sensible et collectif en groupe dans une certaine vision du territoire ${ }^{9}$ ? 


\section{Présentation des projets « Les Promenades blanches » et «Attention à la marche! »}

Dans cette partie, je vais expliciter le cadre et le déroulement des projets en question. Le premier projet s'intitule «Les Promenades blanches». Conçu par l'artiste Mathias Poisson et le chorégraphe Alain Michard en 2006, le projet a été proposé dans le cadre du festival Primavera - Jours de danse, qui a lieu du 10 au 13 mai et est tenu par le Centre chorégraphique national de Nantes (CCNN). Selon l'annonce sur le site du CCNN, l'édition de 2017 « vous embarque pour des promenades, des explorations insolites et en mouvement de l'espace public ", c'est un moment de "partage autour de la danse, ouvert sur la ville ${ }^{10} »$. Dans ce cadre, deux promenades étaient proposées par les artistes, les 12 et 13 mai. Elles ont débuté au centre culturel Cosmopolis, et ont fini au $\mathrm{CCN}$ de Nantes, dans le studio. Une trentaine de personnes ont participé à chaque promenade.

Outre la volonté de proposer une exploration de la ville par le mouvement, il y avait l'intention du CCNN d'ouvrir ses activités à des publics larges, d'aller au-delà de son lieu physique qui est plutôt un lieu de travail ${ }^{11}$. Les organisateurs du festival voulaient aussi créer la connexion entre le Centre et le quartier qui l'entourait. Au début, la promenade devait se faire dans le quartier autour du CCNN. Elle a été finalement étendue à toute la ville, en lien avec les manières dont les artistes ont procédé dans leur choix du parcours. Nous pouvons donc identifier l'intention institutionnelle de proposer une expérimentation collective à un public, sur une partie de la ville. Cette intention convoque un protocole qui met les sujets en mouvement et dans un mode de relation particulier avec le monde.

La promenade commençait par une annonce et une mise en scène du protocole. Alain Michard et Mathias Poisson se sont mis en binôme pour animer le protocole qu'une voix enregistrée prononçait. La présentation du protocole achevée, les personnes se sont réparties en binômes. Une personne portait les lunettes floues, et l'autre la guidait. $\mathrm{Au}$ milieu du parcours, la personne guidée partait pour une courte exploration personnelle, surveillée par son guide. Puis, les rôles s'inversaient. Le temps de la promenade était d'environ 45-50 minutes pour chacun, et d'une durée totale d'environ 2 heures.

La particularité de ce projet doit être soulignée. Elle relève d'abord des manières d'être en binôme. En même temps, l'expérience à deux s'inscrit dans la dimension collective qui est pensée par le protocole et vécue lors de la marche. Les binômes font partie d'un groupe qui est réfléchi au stade de la conception du projet. Par exemple, les manières de se déplacer et les formes que prend le mouvement du groupe par rapport aux lieux traversés, le nombre de participants, le choix des endroits constituent l'objet du travail des artistes. Mathias Poisson m'a parlé de l'évolution du projet depuis sa création en 2006 et de l'introduction des figures chorégraphiques, telle que le cercle, le rapprochement, l'alignement dans le trajet, comme une manière de faire une «forme de groupe ${ }^{12}$ ». 
Figure 1. «Promenades blanches », Rennes, Centre culturel Colombier

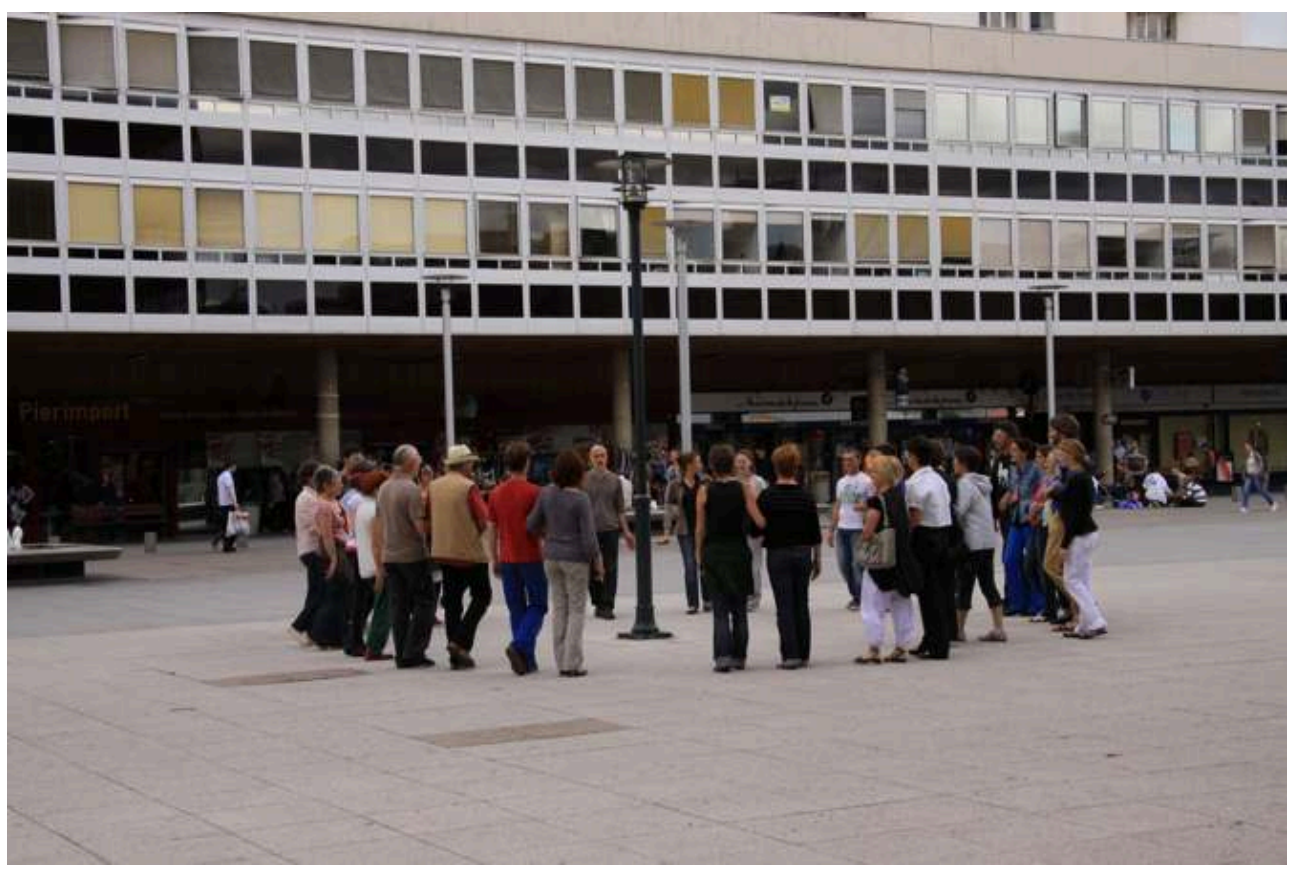

Photo Mathieu Harel-Vivier, source : http://www.alainmichard.org/Promenades-blanches.

17 Ici, un groupe de marcheurs se met en cercle autour d'un lampadaire, dans un mouvement lent, dans un espace vaste et dégagé du centre-ville. Ce mouvement instaure plusieurs dimensions. C'est un moment où l'aspect de marche en binôme s'élargit à quelque chose qui est partagé collectivement à plusieurs niveaux : par la forme du mouvement qui est en même temps inspirée par l'espace environnant, par la présence aux autres dans le groupe, par le rythme que prend le mouvement. Cela permet de penser la question de la présence à plusieurs dans le paysage urbain qui a une incidence sur la forme du mouvement. Cette dernière est-elle suffisante pour créer une relation de groupe, puis une expérience collective ? J'ai évoqué plus haut la volonté du commanditaire du projet de proposer une expérimentation pour un groupe qui se repose sur les protocoles des artistes. Cette volonté a une visée non seulement esthétique, mais aussi territoriale, à l'échelle de la ville. Dans ce sens-là, il me semble possible de convoquer mon hypothèse sur le lien entre le paysage et le territoire et la place de l'expérience dans la vision territoriale. L'expérience sensible et collective, avec le paysage, se traduit dans des manières d'être ensemble sur une temporalité du projet dans un territoire donné. Dans leurs manières de configurer l'expérience, les qualités $\mathrm{du}$ paysage urbain environnant sont prises en compte par les artistes. Puis, les expériences vécues par les participants et la présence collective produisent ce paysage en retour. Comment donc le territoire se construit-il avec cette présence?

Pour continuer et donner une autre piste pour une exploration du lien entre le paysage et l'expérience collective, il faudra mentionner la manière dont la promenade s'ajuste au fur et à mesure du parcours. Mathias Poisson m'a parlé de l'importance du guide volant qui suit la promenade, et qui « [...] observe tout le groupe et [...] fait remonter l'information au premier guide, celui qui est devant. [...] C'est lui qui va décider du rythme, des figures, comment il les fait. [...] On a tout un langage de signes. Ou bien s'il faut ralentir parce qu'il y a des gens fatigués ou s'il faut accélérer pour les rendre un peu joyeux. Donc, c'est une manière de faire du groupe ${ }^{13} »$. 
19 Cette expérience se pense donc au préalable. En même temps, il y aurait une coconstruction de l'expérience dans l'immédiat, pour et avec le groupe, en fonction du mouvement du groupe, des ressentis corporels, des sensations, des indices de différentes natures. Je suggérerais que la notion de correspondance ingoldienne soit applicable pour caractériser ce type de rapport qui s'instaure dans un mouvement réciproque et dynamique. Ce rapport serait aussi celui qui se compose avec le paysage environnant, les lieux traversés, et qui produit des qualités de présence particulières avec l'espace et les autres, sur le territoire tel qu'il est envisagé par ce projet concret.

20 Avec une durée de deux jours et presque $30 \mathrm{~km}$ parcourus, le projet «Attention à la marche! à Bordeaux s'inscrivait dans le programme intitulé "Été métropolitain ${ }^{14}$. Une saison buissonnière " organisé par la métropole bordelaise du 8 juillet au 15 septembre 2017. Selon Clotilde Pascaud, une commanditaire du projet et chef de projets " grands événements, équipements métropolitains et refuges périurbains de la métropole", cette manifestation symbolisait l'ouverture de ce programme. La plaquette indiquait que l'été était « dédié à la (re)découverte de paysages merveilleux, enchanteurs, insolites, atypiques, fascinants sur lesquels nous sommes invités à poser un regard neuf par le truchement de l'art, un art protéiforme qui réenchante le quotidien ${ }^{15}$ ». Ce festival a alors débuté avec "Attention à la marche! ", coproduit par Le Bruit du frigo et le Bureau des guides ${ }^{16}$. Le sous-titre du projet était "Première université d'été de la randonnée périurbaine, autour des refuges périurbains ». Les artistes participant étaient Mathias Poisson, Robin Decourcy, Laurent Petit et le collectif La Folie Kilomètre. Il s'agissait d'une randonnée de deux jours dans la métropole bordelaise, entre Pessac et Floirac, avec une nuit en bivouac à Bègles. Le jour de départ, la randonnée comptait autour de 130 marcheurs sur le campus de l'université de Bordeaux-Montaigne, et s'est achevée à Floirac.

21 Selon Yvan Detraz, le directeur artistique du Bruit du frigo, le parcours réalisé faisait partie des randonnées de l'association et était également articulé autour de trois refuges périurbains (Le Hamac, Les Guetteurs, La Belle étoile), selon le cahier des charges. En ce qui concernait les refuges, l'idée était de les présenter non seulement comme des cabanes où les gens peuvent venir séjourner une nuit, mais aussi comme points qui peuvent servir de repères, être reliés, créer la mobilité ${ }^{17}$. Pour les commanditaires, il était important d'expérimenter le territoire bordelais de manière peu commune, y compris de passer par les endroits qui ne font pas partie des itinéraires souvent choisis par les Bordelais. Il était aussi question de faire marcher les gens des deux côtés de la Garonne, la rive gauche le premier jour et la rive droite le deuxième, avec un accent mis sur cette dernière, du fait que celle-ci était encore mal connue, plus naturelle ${ }^{18}$. L'intention était de donner un aperçu varié du territoire (les paysages, les ambiances, les lieux), afin d'apporter une richesse aux participants et aux propositions artistiques et avec une diversité rythmant le parcours ${ }^{19}$.

22 La métropole a donné carte blanche au Bureau des guides quant au choix des artistes. Le bureau a cherché à introduire la dimension artistique, plastique dans la découverte de différents paysages. Il y avait l'idée d'associer, à la dimension exploratoire proposée par les randonnées du Bruit du frigo, un aspect qui montrait que l'expérimentation du territoire pouvait se faire à travers les différentes manières de marcher, allant au-delà $\mathrm{du}$ simple déplacement ${ }^{20}$. D'après Julie de Muer, membre du Bureau des guides et responsable du commissariat artistique du projet, la question se posait ainsi au départ : «On n'est pas sur notre territoire, comment procède-t-on?» Et la réponse fut de 
l'approcher par les questions du corps et de la perception. Ce questionnement a défini la composition de l'équipe artistique ${ }^{21}$.

Nous pouvons donc voir qu'il y a une considération territoriale en jeu, qui s'inscrit dans le projet plus large de valorisation du territoire qui est déjà en cours (comme les refuges périurbains et les activités qui y sont liées). Le projet qui engage la dimension esthétique provenant des pratiques des artistes et les expériences visées pour les participants renforce le lien entre expérience du paysage et territoire, comme j'en ai émis l'hypothèse. Toutefois, quelques différences peuvent être identifiées avec le projet "Promenades blanches ", suite à la durée et à la longueur du parcours, au territoire parcouru, au nombre de participants plus élevé, aux expériences proposées. Ces aspects interrogent la question de la relation au paysage, mais aussi celle de la nature de l'expérience du paysage traversé sur le plan de la spatialité. Suite à la définition de cette dernière comme unité du sentir et du se mouvoir, et comme une présence particulière au monde, de quelle manière surgit-elle dans un groupe de 130 personnes ? Sur le plan collectif, y a-t-il toujours cette présence? Et comment l'espace traversé prend place dans cette expérience collective?

\section{Méthode et outils de recherche. Première analyse de la matière}

Dans les deux projets et le développement de ma méthode de terrain, j'ai choisi la participation directe. Puisqu'il s'agit d'une expérience qui est tout d'abord individuelle, ma propre expérience et son analyse m'ont paru des outils importants de la recherche. La préparation préliminaire concernait la prise de contact avec les artistes, l'identification des projets, le recueil des informations. En termes d'expérience immédiate, le fait de connaître au préalable le projet et les expériences possibles établissait certaines limites. Pour remédier à cet aspect, un travail de mise en état a été effectué, notamment par l'élaboration de mon protocole de participation. Les consignes que je me donnais visaient à me rendre disponible aux choses qui devaient advenir, à être ouverte aux autres, à ne pas préfigurer ce qui se produirait ${ }^{22}$. Une question se pose - comment se rendre compte du fait que nous nous retrouvons vraiment dans le mode d'être qui correspond à celui du sentir, pour moi-même et ensuite pour les autres? Comment donner forme aux sensations, aux ressentis corporels, physiques, kinesthésiques, à l'imaginaire d'une personne concrète ? La même chose arrive-t-elle aux autres? Et dans un groupe de marcheurs? Existe-t-il un pressenti de l'écart entre ce qui est vécu et son compte rendu ? Le passage au langage consistait en l'écriture du cahier de bord sous la forme du récit de ma propre expérience avant, pendant (parfois) et après la participation. Ce récit intégrait la phase préparatoire de participation (protocole, préparation) et la description de l'expérience vécue. Il a permis d'identifier des éléments de l'expérience qui était la mienne et ceux qui pouvaient potentiellement être présents dans l'expérience des autres.

En m'appuyant sur l'indication de Straus selon laquelle l'expérience vécue n'existe que "pour moi ", l'interrogation de ce qu'elle est pour autrui s'imposait. J'ai donc effectué quelques entretiens avec les participants des projets afin de m'approcher de l'aspect collectif de l'expérience. Je leur ai posé des questions sur leurs états de corps (ressentis sensoriels, physiques) ; leur rapport à autrui et au groupe. La question suivante se pose toutefois : puisqu'il s'agit du vécu d'ordre subjectif, à quel point la personne peut-elle 
exprimer ce qui lui est arrivé ? Et comment donc analyser ces dires, qui concernent peut-être une intuition, un présupposé, une émotion ${ }^{23}$ ? Une autre contrainte était de ne pas influencer ces réponses par mon positionnement de chercheuse et de ne pas les diriger là où mes hypothèses m'amènent. J'ai essayé de laisser dire, laisser revenir l'expérience à travers la parole. Il m'a semblé que dans l'expérience de ces deux projets, la mienne ou celle des autres, certains éléments de l'expérience peuvent être comparés. Je voudrais donc m'attarder sur quelques éléments qui permettent de caractériser ce vécu du point de vue de la spatialité et de l'expérience individuelle, mais aussi potentiellement du point de vue de la relation avec les autres, l'espace environnant et le paysage.

Lors des marches, la question du sol et de la relation au sol, aux surfaces, au revêtement, était importante. À Nantes et à Bordeaux, cela a commencé avec le premier pas que j'ai fait. Tenue par ma partenaire pendant les "Promenades blanches " à Nantes, les surfaces ressenties et le poids des pas étaient quelquefois atténués. Pendant la marche de deux jours à Bordeaux, la sensation de mes propres pieds, et puis celle de leur contact avec les sols étaient plus prononcées. De même, ma partenaire de Nantes m'a parlé du sol et de sa qualité : « Moi j'ai remarqué que, [...], à partir du moment où le sol il est droit, c'est plus simple, parce que là j'étais sûre, et quand il y a des escaliers ou ça descend, [...] ça devient plus difficile ${ }^{24}$.» Un participant de Bordeaux m’a parlé du fait de marcher sur le bitume : «En général, les moments les plus vivifiants pour moi étaient ceux où l'on marchait dans les bois, et les plus fatigants, ceux où l'on marchait sur le bitume ${ }^{25}$. " Ces ressentis posent la question de la qualité du sol et du lien entre cette qualité et la relation au paysage. La question de la relation au sol serait également liée à la notion de gravité qui se déploie entre la terre et le ciel :

« D’abord la gravité, le perçu pondéral, est-ce que nous ressentons le poids de notre corps de haut en bas? Ce qui dans un second mouvement nous permettra d'être gravide, donner sens à une deuxième direction opposée [...]. Ce deuxième mouvement vers le haut gardera toujours la mémoire du premier qui lui a donné naissance. » (Godard, 1992.)

La gravité, dans son attirance à la fois vers le haut et vers le bas, et la relation au sol nous relient à l'horizon, et ceci informe potentiellement notre expérience immédiate et les manières d'être au monde et avec les autres. Selon Catherine Grout, la notion de l'horizon exprime «[...] cet engagement pour et avec le paysage qui est associé non pas (d'abord ou seulement) au regard mais à la relation sensorimotrice (aller vers et s'éloigner) à partir du contact avec le sol et dans le croisement des axes horizontal (bassin) et vertical (colonne vertébrale et posture érigée). [...] l'horizon comme structure fondatrice de la relation au paysage comme spatialité » (Grout, 2018).

La relation à l'horizon influence nos manières d'être avec le monde et potentiellement avec les autres dans la dimension du sentir, elle agit sur les manières de se mouvoir. Par exemple, une des participantes de Nantes a fait le lien entre le caractère ouvert de l'espace et son envie d'ouverture vers lui : "C'est surtout quand nous sommes entrés dans le parc avec le grand espace de verdure et de silence que je me suis rendu compte de l'espace ouvert devant nous et à quel point ça m'a fait du bien de le percevoir. J'avais notamment envie de faire de grands mouvements ou de grandes respirations ${ }^{26}$.» 
Figure 2. «Attention à la marche ! », Bordeaux, 8 au 9 juillet 2017

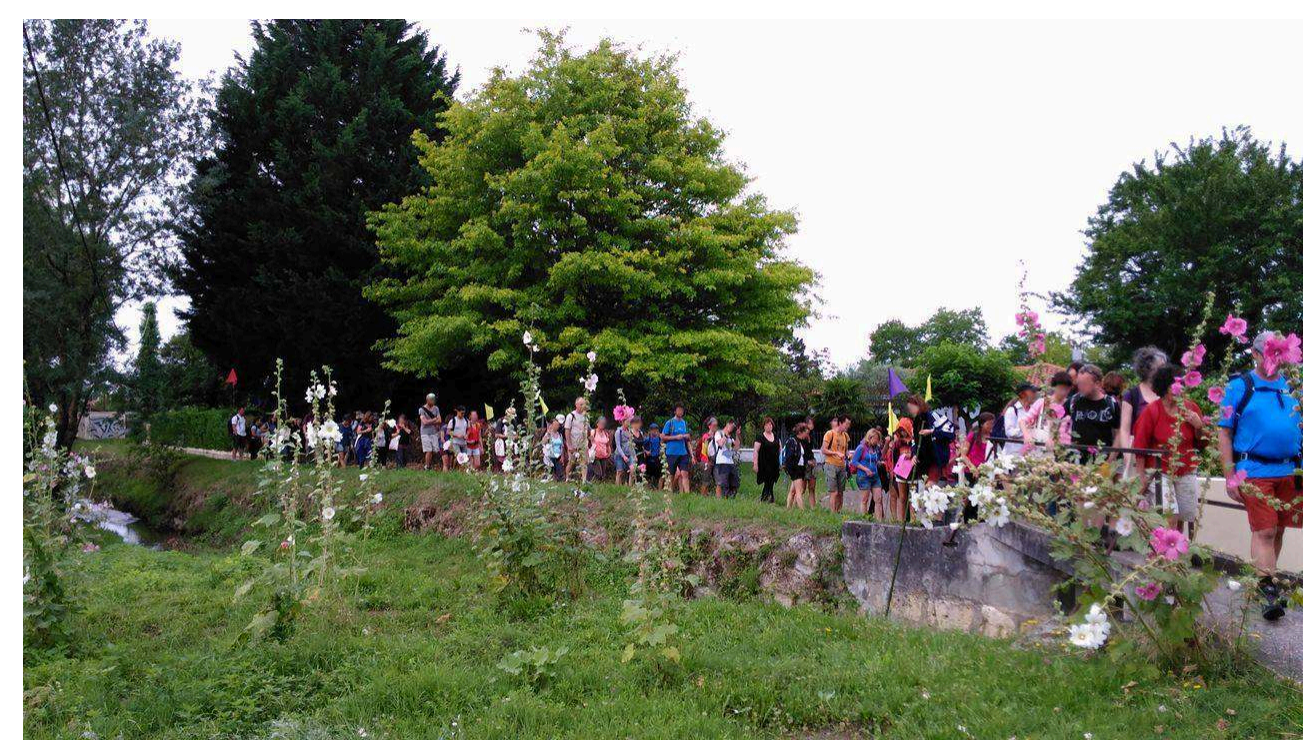

Photo d'Ekaterina Shamova, le 9 juillet 2017 (les visages ont été floutés).

Pour continuer sur ce point, le dialogue entre l'espace environnant et les manières de s'y mouvoir individuellement peut potentiellement sous-tendre les manières de se déplacer ensemble, en rapport avec le paysage. Dans la photo ci-dessus, nous voyons une partie de participants du projet à Bordeaux. Un chemin bordé par un canal d'un côté et par la végétation de l'autre, plus loin, fait que les personnes marchent plutôt en file indienne, lentement, empruntant ce chemin étroit en gravier et un petit pont. Le mouvement du groupe dialogue avec les qualités du paysage autour. Cette réflexion renvoie également à mon interrogation sur la forme du mouvement (comme dans le cas du projet « Promenades blanches ») et à l'expérience immédiate que celle-ci induit sur le plan à la fois individuel et collectif.

ost aussi à comprendre au-delà du sens spatio-temporel, du visible. Toujours selon Catherine Grout, l'horizon est une «structure ouverte et dynamique " qui est ressentie «dans la tension de deux pôles (terre/ciel) qui participent dans ma verticalisation avec l'attirance gravitaire et l'appel vers le ciel » et lié au mouvement. Il existe à la fois autour et à l'intérieur du sujet sentant et cela ne devient possible que si la personne est ouverte et orientée vers le monde (Grout, 2012, p. 27). Cette vision de l'horizon comme quelque chose qui permet de s'ouvrir au monde en laissant place à l'imprévisible et au non qualifiable me semble particulièrement propice à l'analyse de l'expérience des projets en question. Il s'agit d'un champ d'expériences élargi qui fait que le sujet s'ouvre au rapport dynamique avec le monde. Je retrouve cette piste dans la phrase suivante de Tim Ingold sur les manières de se déplacer:

31 «Le voyageur itinérant adapte constamment son mouvement - son orientation et son rythme - à ce qu'il perçoit de l'environnement en train de se découvrir sur son chemin. Il observe, écoute, et ressent ce qui se passe, tout son corps est sur le qui-vive, attentif aux innombrables indices qui, à tout moment, peuvent l'inciter à modifier sa position. » (Ingold, 2011, p. 105.)

Une telle définition d'un rapport à l'itinérance est proche de ce qui est décrit par Julie Perrin sur le projet «Promenades blanches » en tant qu' « expansion sensible », comme réponse à une sollicitation du monde : «Il s'agit de perdre ses repères et orientations 
géographiques pour inventer une autre façon de se situer, non plus géométriquement, mais dans une forme d'expansion sensible, sans destination. » (Perrin 2016, p. 30.) Je parle ici d'une expérience qui se déploie dans la multitude des sensations et dans des rapports variés qui se forment lors du parcours : le rapport au sol, au ciel, le rapport aux ambiances environnantes, aux espaces traversés, à son partenaire, aux autres marcheurs. Cette expansion est aussi imaginaire et non envisageable. Dans le cas du projet "Promenades blanches", par exemple, ces différents niveaux de l'expérience viennent en lien avec ce que Mathias Poisson appelle « un espace flottant». Ce dernier " s'ouvre à celui qui prend le temps d'observer ce qu'il ressent dans un lieu en utilisant tous les sens qu'il possède. Prendre le temps de laisser l'imaginaire réagir aux sensations» (Poisson, 2017.) Cet espace «flou» dont parle l'artiste pourrait correspondre au rapport au monde dans le sentir, qui ne chercherait pas à nommer ou à expliquer les ressentis, le visible, le tangible, mais plutôt d'être là au moment où nous sommes, être au présent, avec les autres et avec le monde environnant.

\section{Conclusion}

Dans ce texte j'ai mis en avant le début de l'analyse des expériences de deux projets, «Promenades blanches » et "Attention à la marche!». Les prémisses ont été posées quant aux appuis théoriques qui permettent de donner forme aux vécus, aux ressentis immédiats, et de les inscrire dans les paysages où ils se déroulent. Les différents éléments de l'expérience (rapport au sol, à l'horizon, la gravité, la relation à autrui, les dimensions variables des expériences) seront à complémenter avec d'autres éléments qui surgiront au fur et à mesure de l'analyse approfondie des projets. Ces expériences seront à situer dans une perspective plus large de convergence entre le vécu individuel et collectif et son lien avec le territoire parcouru et la dimension institutionnelle et conceptuelle des projets (les intentions des commanditaires, la visée pour le territoire, les protocoles artistiques, les manières d'aborder le groupe). J'ai proposé une articulation entre les manières de vivre le paysage et les façons de construire un territoire à travers un projet artistique convoqué par une institution. Ce présupposé semble soutenir certaines intentions de commanditaires et d'artistes pour les projets évoqués qui se traduisent dans les expériences concrètes vécues à la fois individuellement et en groupe. Les questions à poursuivre seront: Comment construirons-nous le territoire, à travers le mouvement, le déplacement? Quelle en sera la part de l'expérience immédiate du paysage ? Et comment la marche à plusieurs informera-t-elle cette transformation?

Je tiens à remercier ma directrice de thèse Catherine Grout pour sa lecture minutieuse de cet article et pour ses conseils pertinents qui ont enrichi ma réflexion. 


\section{BIBLIOGRAPHIE}

Besse, J.-M., « Les cinq portes du paysage. Essai d'une cartographie des problématiques paysagères contemporaines ", Le Goût du monde. Exercices de paysage, Arles, Actes Sud, 2009, p. 15-69.

Besse, J.-M., « Quatre notes conjointes sur l'introduction de l'hodologie dans la pensée contemporaine ", dans Carnets du paysage. Cheminements, Arles/Versailles, Actes Sud/École nationale supérieure de paysage de Versailles, n 11, 2004, p. 26-33.

Careri, F., Walkscapes : Walking as an Aesthetic Practice, Barcelone, Gustavo Gili, 2002, 203 p.

Davila, T., Marcher, Créer. Déplacements, flâneries, dérives dans l'art de la fin du XXe siècle, Paris, Éditions du Regard, 2002, 192 p.

Descola, P. et Ingold, T., Être au monde. Quelle expérience commune ?, débat présenté par Michel Lussault, Lyon, Presses universitaires de Lyon, 2014, 75 p.

Godard, H., « Présentation d'un modèle de lecture du corps en danse », dans Arguel, M. (dir.), Le Corps en jeu, Paris, PUF, 1992.

Grout, C., «Le sentir : au fondement du politique Pour une considération de l'horizon et d'un sol commun ", Projets de paysage, n 18, juillet 2018, URL : http://www.projetsdepaysage.fr/ le_sentir_au_fondement_du_politique.

Grout, C., L'Horizon du sujet. De l'expérience au partage de l'espace, Bruxelles, La Lettre Volée, 2012, $120 \mathrm{p}$.

Ingold, T., « La vie dans un monde sans objets », Perspective, vol. 1, 2016, mis en ligne en décembre 2017, URL : http://journals.openedition.org/perspective/6255.

Ingold, T., The Making. Anthropology, Archaeology, Art and Architecture, London, Routledge, 2013, $164 \mathrm{p}$.

Ingold, T., Une brève histoire des lignes, traduit de l'anglais par Sophie Renaut, Zones Sensibles, 2011, 251 p.

Merleau-Ponty, M., La Phénoménologie de la perception, Paris, Gallimard, 1995, 537 p.

Perrin, J., « Traverser la ville ininterrompue : sentir et se figurer à l'aveugle. À propos de Walk, Hands, Eyes (a city) de Myriam Lefkowitz », Ambiances, 2017, URL : https:// journals.openedition.org/ambiances/962.

Perrin, J., « Sensibilités hodologiques », dans Després, A. (dir.), Gestes en éclats : art, danse et performance, Dijon, Les Presses du réel, 2016, p. 28-38.

Poisson, M., « Marcher à la vitesse d'homme », Nectart, n² 4, 2017.

Straus, E., Du sens des sens. Contribution à l'étude des fondements de la psychologie (1935), traduit de l'allemand par G. Thines et J.-P. Legrand, Grenoble, Éditions Jérôme Millon, 2000, 477 p.

Tiberghien, G., " Hodologique », dans Carnets du paysage. Cheminements, Arles/Versailles, Actes Sud/École nationale supérieure de paysage de Versailles, n 11, 2004, p. 7-25. 


\section{NOTES}

1. Voir http://www.bruitdufrigo.com/index.php?id=356, consulté le 7 juillet 2017.

2. Nous pouvons citer ici l'ouvrage de Thierry Davila Marcher, créer. Déplacements, flâneries, dérives dans l'art de la fin du XX $X^{e}$ siècle (2002) ou Walkscapes. Walking as an aesthetic practice (2002) de Francesco Careri, qui étudient la marche comme une pratique esthétique et comme un outil de la création plastique.

3. Journées d'études "Les promenades sonores et chorégraphiques en question", 12-13 janvier 2018, CND Pantin. Annonce des journées d'études, consulté le 10 janvier 2018 sur http:// www.labex-arts-h2h.fr/les-promenades-sonores-et.html?lang=fr.

4. Je parlerai de façon plus extensive de la portée politique des projets dans la phase ultérieure de ma recherche. L'intention politique se révélera à plusieurs niveaux. D'abord proposer une expérience collective avec une certaine visée (par un organisme d'accueil), qui peut être effectuée grâce à un protocole particulier d'un artiste qui prévoit une certaine configuration de l'expérience sur le plan individuel et collectif. Puis, l'expérience vécue au sein du projet permettra de faire dialoguer toutes ces dimensions. L'étude compréhensive est envisagée afin d'identifier les différences, les lignes de comparaison ou les complémentarités entre les projets de mon corpus et les manières dont la question du politique y apparaît.

5. La première édition en langue allemande datant de 1935 , je me réfère à la $2^{\mathrm{e}}$ édition de la traduction française de cet ouvrage par Georges Thines et Jean-Pierre Legrand (2000).

6. Je souligne. La différence entre "mon monde » et « le monde » est à noter dans la pensée de Straus. Toutefois, cette analyse ne sera pas développée dans ce texte.

7. Traduit par l'auteur.

8. La question de la marche serait aussi à traiter à travers la pensée sur l'espace hodologique, en lien avec les qualités attribuées à ce processus par le psychologue allemand Kurt Lewin. Je renvoie ici aux articles suivants : Tiberghien, G., « Hodologique » (2004, p. 7-25), et Besse, J-M., « Quatre notes conjointes sur l'introduction de l'hodologie dans la pensée contemporaine » (2004, p. 26-33).

9. La notion du « bien vivre ensemble » m'intéresse également dans la pensée de Jean-Marc Besse sur le paysage : «Le paysage, [...] est l'expression d'une interrogation à propos du bien-être ou du "bien vivre ensemble" des communautés humaines, il incarne une interrogation sur les valeurs qui peuvent fonder ce "bien vivre ensemble"»(Besse, 2009, p. 39). Je voudrais par la suite analyser les projets sur lesquels je travaille à partir de cette notion. Y-aurait-il un sous-entendu d'une telle « interrogation »?

10. Site du CCNN Nantes, http://ccnnantes.fr/, consulté le 5 mai 2017.

11. Conversation avec Erika Hess, directrice déléguée du CCNN Nantes, le 10 septembre 2018.

12. Entretien avec Mathias Poisson, Marseille, le 31 mai 2018.

13. Ibid.

14. Été métropolitain. Une saison buissonnière, plaquette de l'événement, Bordeaux Métropole, 2017, $40 \mathrm{p}$.

15. Juppé, A., "Édito ", Été métropolitain. Une saison buissonnière, plaquette de l'événement, Bordeaux Métropole, 2017, p. 2.

16. Conversation téléphonique avec Clotilde Pascaud, le 5 avril 2018. Initialement la métropole a sollicité le Bureau des guides qui, à leur tour, se sont adressés au Bruit du frigo, qui sont ancrés dans le territoire et sont également à l'origine des Refuges périurbains.

17. Ibid.

18. Ibid. Selon Clotilde Pascaud, le but n'était pas de faire voir les paysages dits « beaux », mais de faire une découverte « exhaustive » du territoire.

19. Conversation téléphonique avec Yvan Detraz, le directeur artistique du Bruit du frigo, le 25 septembre 2017. 
20. Conversation téléphonique avec Clotilde Pascaud, le 5 avril 2018.

21. Conversation téléphonique avec Julie de Muer, membre du Bureau des guides, le 5 octobre 2017.

22. Pour ces consignes, je renvoie au séminaire doctoral du laboratoire LACTH (ENSAP Lille) qui s'intitule "Spatialité et situations ", le 5 mars 2014, et notamment au texte de Catherine Grout "Spatialité, situation, description" sur les points de méthode qu'elle propose, p 12-13, URL: http://www.lille.archi.fr/ressources/20580/72/lacth_crsemdoct5mars14_spatialit_.pdf.

23. La différence est à noter entre les entretiens in vivo et par mail. Dans les premiers, il y a la proximité immédiate avec ce qui a été vécu. Dans l'écrit, il y a une possibilité de prendre du recul sur le vécu. Ou à l'inverse, les réponses sont raccourcies et très brèves. Je me rends compte de la distance qui pourrait exister par rapport à la chercheuse. Pour exploiter ces entretiens et analyser le dit et le non-dit, les hésitations, les omissions, je voudrais recourir aux outils de l'analyse du discours dans la phase ultérieure de l'analyse de ces paroles.

24. Entretien effectué à Nantes, le 12 mai 2017.

25. Entretien effectué par mail après le projet « Attention à la marche ! », le 27 juillet 2017.

26. Entretien avec une participante du projet à Nantes, par mail, mai 2017.

\section{RÉSUMÉS}

Cet article présente une recherche en cours sur l'expérience individuelle et collective au sein des projets artistiques de marche collective dans le paysage. Ces projets interrogent le lien entre un vécu personnel et collectif et le rôle de la marche dans cette présence au monde collective. En partant du corpus recueilli et de son analyse, nous tentons de comprendre la nature de ce qui surgit dans un groupe de marcheurs. Le lien que cette expérience entretient avec le paysage traversé donne une certaine vision des présences collectives sur un territoire. Le point de départ est la notion de la spatialité en tant qu'unité du sentir et du se mouvoir qui reflète l'expérience immédiate du participant à ces projets. Toutefois, en supposant qu'une telle expérience soit d'ordre subjectif, les manières dont une expérience collective advient sont questionnées.

This article presents an ongoing study on the individual and collective experience in artistic projects involving group walks in landscapes. These projects question the relationship between the individual and group experience and the role of the walk in a collective context. From the material gathered and studied, the objective is to try to understand the nature of what emerges among a group during a walk. The relationship such an experience establishes with the landscape the group walks through provides a view of collective presences in a given area. The point of departure is the notion that spatiality may be considered in terms of a unity of sensing and movement reflecting the experience of the participants in these projects. However, assuming that such an experience is subjective, the different manners in which a collective experience occurs are also considered.

INDEX

Mots-clés : spatialité, expérience, mouvement, marche, paysage

Keywords : spatiality, experience, movement, walk, landscape 


\section{AUTEUR}

\section{EKATERINA SHAMOVA}

Ekaterina Shamova, doctorante en $2^{\mathrm{e}}$ année au laboratoire LACTH à l'École nationale supérieure d'architecture et de paysage de Lille (ED SHS Lille3), elle écrit sa thèse sous la direction de Catherine Grout qui porte sur les projets artistiques de marche collective.

e-shamova[at]lille.archi[dot]fr 